\title{
MATHEMATICAL REFORMULATION OF THE KOLMOGOROV-RICHARDSON ENERGY CASCADE IN TERMS OF VORTEX STRETCHING
}

\author{
TSUYOSHI YONEDA, SUSUMU GOTO, AND TOMONORI TSURUHASHI
}

\begin{abstract}
In this paper, with the aid of direct numerical simulations (DNS) of forced turbulence in a periodic domain, we mathematically reformulate the Kolmogorov-Richardson energy cascade in terms of vortex stretching. By using the description, we prove that if the Navier-Stokes flow satisfies a new regularity criterion in terms of the enstrophy production rate, then the flow does not blow up. Our DNS results seem to support this regularity criterion. Next, we mathematically construct the hierarchy of tubular vortices, which is statistically self-similar in the inertial range. Under the assumptions of the scale-locally of the vortex stretching/compressing (i.e. energy cascade) process and the statistical independence between vortices that are not directly stretched or compressed, we can derive the $-5 / 3$ power law of the energy spectrum of statistically stationary turbulence without directly using the Kolmogorov hypotheses.
\end{abstract}

\section{INTRODUCTION}

Since the seminal study by Orszag and Patterson (1972) [20, the numerical integration (i.e. the direct numerical simulations, DNS) of the Navier-Stokes equation without any turbulence model has been playing important roles in turbulence researches; see Ref. 11 for a review of DNS of turbulence. One of the most important features unveiled by DNS of turbulence is that turbulence is not random but composed of coherent motions. In particular, recent DNS [7, 9, 17, 18, of turbulence at sufficiently high Reynolds numbers have revealed that there exists a hierarchy of coherent vortices in developed turbulence.

In the present study, we investigate the energy cascade picture [23, 5] in turbulence of an incompressible fluid with uniform density and kinematic viscosity $\nu$ under periodic boundary conditions in three orthogonal directions; and, on the basis of the picture, we develop arguments on the global-in-time smooth solution of the Navier-Stokes equation. Since we need external force to drive flow in a periodic cube, we impose body force $f$ which injects the kinetic energy at a large scale $\mathcal{L}$. Here, we define the Reynolds number by

$$
R e=U \mathcal{L} / \nu
$$

with $U$ being the characteristic velocity directly driven by $f$. When $R e$ is sufficiently high (more concretely, higher than $O\left(10^{4}\right)$ [4]), turbulence is composed of vortices with various length scales. We reemphasize that these vortices are coherent

Date: December 9, 2021.

2020 Mathematics Subject Classification. Primary 76F02; Secondary 76F65; Tertiary 35Q30; Quaternary 76D03. 
in time and space. The hierarchy of coherent vortices and its sustaining mechanism of this statistically stationary turbulence in a periodic cube are rather simple 9 . If we visualize vortices by using the magnitude of vortices, we can only observe the forest of the smallest-scale vortices (Fig. 1 in Ref. [11]). This is because the vorticity is predominantly determined by the smallest-scale eddies. Therefore, we need a scale decomposition to capture the hierarchy of coherent vortices. More precisely, the band-pass filter (i.e. the Littlewood-Paley decomposition) of the Fourier components of the vorticity reveals a clear hierarchical structures of coherent tubular vortices (see Fig. 2 in Ref. 9 ). The sustaining mechanism of the hierarchy of vortices is also simple. Tubular vortices at a given length (i.e. a given thickness) are stretched and amplified in straining motions around (pairs of) larger tubular vortices. Here, it is important that this generation process of smaller vortices by larger ones occurs locally in scale. More quantitatively, 2-8 times larger vortices stretch and generate vortices at a given scale (see Sec. 2.3 for the present DNS results). In other words, this sustaining process of turbulence is consistent with the classical Kolmogorov theory (i.e. the Kolmogorov-Richardson energy cascade [5]). It is also important that the smallest length scale, namely the Kolmogorov length $\eta$, is determined by the length for which the two time scales of the vortex stretching and viscous effects are balanced. Therefore, the hierarchy of vortices is sustained in the inertial range of the length scales between $\mathcal{L}$ and $\eta$, but vortices cannot be amplified in the viscous dissipation range of the length scales smaller than $\eta$.

The above-mentioned scale locality of the vortex stretching is also consistent with the fact that the strain-rate, $U / \mathcal{L}$, induced by the largest scale, $\mathcal{L}$, flow cannot overcome the viscous effects for the length scales smaller than the Taylor length $\lambda 1$. Note that $\lambda \gg \eta$ for high Reynolds numbers. It is also important that the ratio $\mathcal{L} / \eta$ increases with the Reynolds number as $\mathcal{L} / \eta \sim R e^{3 / 4}$. Hence, physically speaking:

Observation 1.1. The increase of Re with fixing $f$ (i.e. the decrease of $\nu$ with fixing $U$ and $\mathcal{L}$ ) simply adds the number of the levels of the hierarchy of vortices.

In other words, this physical picture may allow us to expect that a finite energy dissipation rate $\epsilon$ can be sustained in the shear flow around thin coherent tubular vortices even in the limit $\nu \rightarrow 0$.

Remark 1. Jeong and the first author [12] mathematically considered the balance between the viscous dissipation and the vortex stretching. More precisely, they prepared small-scale vortex blob and large-scale anti-parallel vortex tubes for the initial data (in other words, shear flows around thin coherent tubular vortices), and showed that the corresponding 3D Navier-Stokes flow creates instantaneous vortexstretching. Using this stretching, they showed that the flows satisfy a modified version of the zeroth law (but very close to the actual one) in a uniform time interval which in particular implies enhanced dissipation. The zeroth law postulates that, under the normalization of the initial data (depending on $\nu)\left\|u_{0}^{\nu}\right\|_{L^{2}}=1$, the mean

\footnotetext{
${ }^{1}$ We consider statistically stationary turbulence and we define length scales $(\mathcal{L}, \lambda$ and $\eta)$ by their averages. By the definition of the Taylor length and the dissipation law [22] for statistically stationary turbulence we obtain $\nu U^{2} / \lambda^{2} \sim \epsilon \sim U^{3} / \mathcal{L}$, which reduces to the balance of the two time scales: $\lambda^{2} / \nu \sim \mathcal{L} / U$. Although it is an important issue to investigate the temporal fluctuations of the energy dissipation rate and these length scales 225, 10, this is out of scope of the present study. Here, we investigate the averaged picture of the energy cascade in a sufficiently large domain.
} 
energy dissipation rate of the corresponding 3D Navier-Stokes flow $u^{\nu}$ does not vanish as $\nu \rightarrow 0$ :

$$
\liminf _{\nu \rightarrow 0} \nu\left\langle\left\langle\left|\nabla u^{\nu}\right|^{2}\right\rangle\right\rangle>0
$$

where $\langle\langle\cdot\rangle\rangle$ denotes some ensemble or long-time, space averages.

The main purpose of the present study is to mathematically describe the feature of solutions of the Navier-Stokes equation on the basis of these physical pictures of turbulence. More concretely, with the aid of the DNS of turbulence in a periodic cube (the details of which are given in Sec. 2), first, we observe that the NavierStokes turbulence is mathematically smooth enough (Sec. 3). Second, we propose a mathematical description of the energy cascading process in developed turbulence (Sec. 4). Then, we derive the Kolmogorov $-5 / 3$ power law of the energy spectrum by employing this energy cascade picture, without directly using the Kolmogorov hypotheses. (Sec. 5).

Notation. We use $A \lesssim B$ (equivalently, $B \gtrsim A$ ) if there is an absolute constant $C>0$ such that $A \leq C B$. Then, we say $A \approx B$ if $A \lesssim B$ and $B \lesssim A$. Moreover, $A \lesssim \gamma B$ means $A \lesssim C_{\gamma} B$ with some constant $C_{\gamma}$ depending on $\gamma$.

\section{NUMERICAL SIMULATIONS}

In this section, we summarize the numerical method and parameters, and we show the results of our DNS.

2.1. Numerical method. We numerically integrate the Navier-Stokes equation in $\mathbb{T}^{3}:=(\mathbb{R} / 2 \pi \mathbb{Z})^{3}$ :

$$
\left\{\begin{array}{r}
\partial_{t} u+u \cdot \nabla u+\nabla p=\nu \Delta u+f, \\
\nabla \cdot u=0, \\
u(t=0)=u_{0}
\end{array}\right.
$$

with the external force,

$$
f=(-\sin x \cos y,+\cos x \sin y, 0) .
$$

Here, $u:[0, \infty) \times \mathbb{T}^{3} \rightarrow \mathbb{R}^{3}$ and $p:[0, \infty) \times \mathbb{T}^{3} \rightarrow \mathbb{R}$ denote the velocity and pressure of the fluid, respectively. We use the standard Fourier spectral method. Here, we numerically integrate, by the fourth order Runge-Kutta-Gill scheme, the vorticity equation in terms of the Fourier component $\widehat{\omega}(K)$ of vorticity, which is defined by

$$
\omega(x)=\sum_{K \in \mathbb{Z}^{3}} \widehat{\omega}(K) e^{i K \cdot x} \quad \text { with } \quad(\mathcal{F} u)(K)=\widehat{u}(K):=\frac{1}{(2 \pi)^{3}} \int_{\mathbb{T}^{3}} u(x) e^{-i K \cdot x} d x .
$$

Note that we need to integrate only two components of $\widehat{\omega}(K)$ because of the solenoidal condition $K \cdot \widehat{\omega}=0$. For numerical efficiency, we use the fast Fourier transform to evaluate the nonlinear term in the vorticity equation in the real space. Here, we remove the aliasing errors by the phase shift method. 


\begin{tabular}{c|cccc} 
Run & $N^{3}$ & $\nu$ & $k_{\max }\langle\eta\rangle$ & $\left\langle R_{\lambda}\right\rangle$ \\
\hline \hline 2-1K & $1024^{3}$ & 0.002 & 3.0 & 296 \\
\hline $8-1 \mathrm{~K}$ & $1024^{3}$ & 0.008 & 9.3 & 123 \\
\hline $8-2 \mathrm{~K}$ & $2048^{3}$ & 0.008 & 20 & 136
\end{tabular}

TABle 1. Numerical parameters. $N^{3}$, the number of the Fourier modes; $\nu$, the kinematic viscosity; $\langle\eta\rangle$, the temporal average of the Kolmogorov length scale $\left(k_{\max }=\sqrt{2} N / 3\right.$ denotes the maximum wavenumber); $\left\langle R_{\lambda}\right\rangle$, the temporal average of the Taylor-length Reynolds number.

2.2. Numerical parameters. Since we fix the external force $f$, we change the Reynolds number by changing $\nu$. We denote the spatial average of the energy dissipation rate by $\epsilon(t)$. Then, the Kolmogorov length scale is expressed by $\eta(t)=$ $\epsilon(t)^{-1 / 4} \nu^{3 / 4}$. We choose the number $N^{3}$ of the Fourier modes so that we can resolve the small-scale structures of size $\eta(t)$. In the numerical simulations of turbulence, we use the indicator of the resolution $k_{\max }\langle\eta\rangle$, where $\langle\eta\rangle$ is the temporal average of $\eta(t)$ and the maximum wavenumber $k_{\max }=\sqrt{2} N / 3$ is the present numerical scheme. Usually, the condition $k_{\max }\langle\eta\rangle>1$ is recommended. In the present study, however, we adopt much larger value (say, $O(10)$; see table 1) to resolve flow structures much smaller than $\langle\eta\rangle$. We also examine that the small-scale statistics are independent of artificial parameters by changing $N^{3}$ with a common value of $\nu$.

We choose the time increment of the numerical integration so that the CFL condition is satisfied. We set the integration time of each run to be longer than the turnover time of the largest eddies. We evaluate the development of turbulence by the Reynolds number

$$
R_{\lambda}(t)=u^{\prime}(t) \lambda(t) / \nu,
$$

where $\lambda$ is the Taylor length and $u^{\prime}(t)$ (i.e. the standard deviation of a velocity component) denotes turbulence intensity. In homogeneous isotropic turbulence,

$$
R_{\lambda}(t)=\sqrt{\frac{20}{3 \nu \epsilon(t)}} \mathcal{E}(t),
$$

where $\mathcal{E}(t)$ is the kinetic energy per unit mass. In general, turbulence is fully developed when $R_{\lambda}>140$ [4. Turbulence with lower $R_{\lambda}$, the scale separation between the largest scale, i.e. the forcing scale, and $\eta$ is insufficient.

In the following, we show our numerical results obtained by the simulation with the parameters given in table 1 .

\subsection{Energy transfer due to vortex stretching.}

2.3.1. Four wavenumber ranges. There are four distinct wavenumber ranges (or equivalently, scale ranges) in each of which the energy transfer due to vortex stretching exhibits different characteristics. To show this, we define the energy transfer due to vortex stretching (see the energy equation (5.2) by

$$
T\left(K, K^{\prime}\right):=\frac{1}{K^{2}} \int_{\mathbb{T}^{3}} \omega(K) \cdot \nabla u\left(K^{\prime}\right) \cdot \omega(K) d x
$$



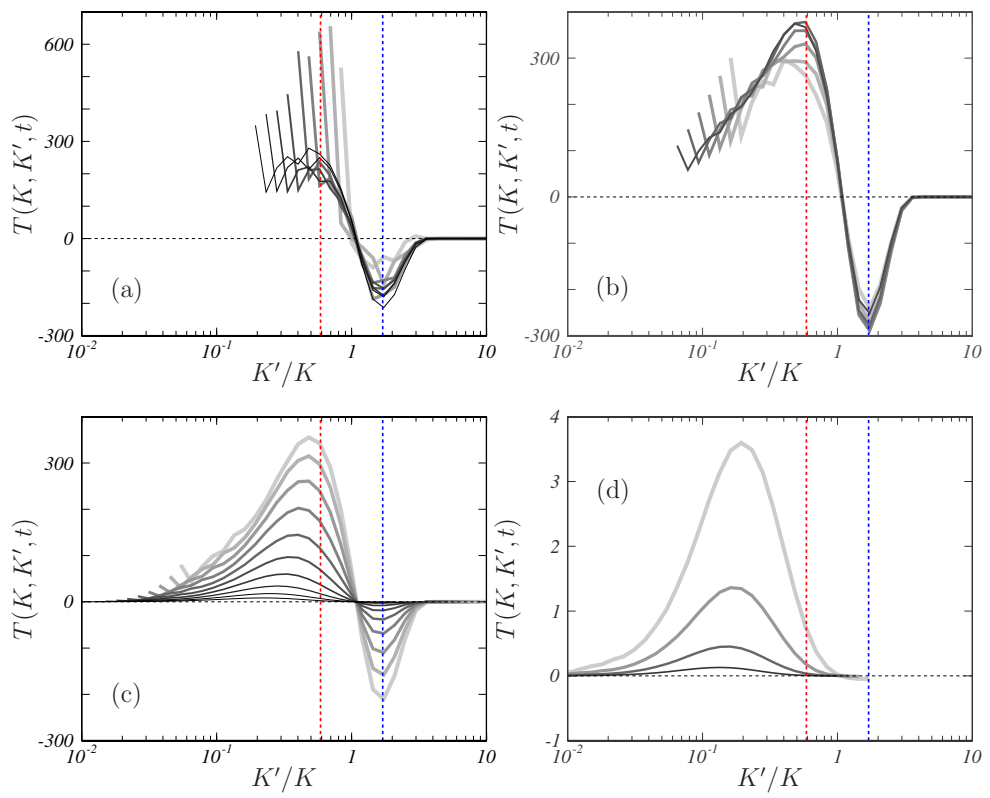

Figure 1. Energy transfer $T\left(K, K^{\prime}, t\right)$ to the Fourier modes at wavenumber $K$ from the Fourier modes at $K^{\prime}$ due to vortex stretching in the four different wavenumber ranges. In each plot, darker (thinner) curves correspond to larger $K$. Red and blue vertical lines indicate $K^{\prime}=0.58 K(=K / 1.7)$ and $K^{\prime}=$ $1.7 K$, respectively. Note that, from this observation, we assume $\alpha=1.7$ in Sec. 4 (a) In the energy containing range $K \in\left[1.2^{-34} \eta^{-1}, 1.2^{-26} \eta^{-1}\right]=\left[0.0020 \eta^{-1}, 0.0087 \eta^{-1}\right]$, where the Fourier modes (which correspond to the whiskers observed at the lower wavenumber) directly driven by external force. (b) In the inertial range $\left(K \in\left[1.2^{-25} \eta^{-1}, 1.2^{-20} \eta^{-1}\right]=\left[0.010 \eta^{-1}, 0.026 \eta^{-1}\right]\right)$, where we can observe a clear collapse of the curves for different $K$, and the peaks are located at $K^{\prime}=0.58 K$ and the valleys are at $K^{\prime}=1.7 K$. (c) In the dissipation range $K \in$ $\left[1.2^{-19} \eta^{-1}, 1.2^{-10} \eta^{-1}\right]=\left[0.031 \eta^{-1}, 0.16 \eta^{-1}\right]$, where we do not observe the self-similarity of the energy transfer. (d) In the far dissipation range $K \in\left[1.2^{-9} \eta^{-1}, 1.2^{-6} \eta^{-1}\right]=\left[0.19 \eta^{-1}, 0.33 \eta^{-1}\right]$, where the energy is dissipated by viscosity and almost no energy is transferred to higher wavenumbers $\left|T\left(K^{\prime}>K\right)\right| \ll\left|T\left(K>K^{\prime}\right)\right|$. Results of Run $2-1 \mathrm{~K}\left(R_{\lambda} \approx 280\right)$.

where

$$
\omega(K):=\mathcal{F}^{-1}\left[\chi_{[K / \sqrt{2}, \sqrt{2} K)} \mathcal{F}[\omega]\right]
$$


and

$$
u(K):=\mathcal{F}^{-1}\left[\chi_{[K / \sqrt{2}, \sqrt{2} K)} \mathcal{F}[u]\right] .
$$

Note that in the next section we will define $\omega^{k}$ by $\omega\left(2^{k}\right)$. We plot $T\left(K, K^{\prime}, t\right)$ in turbulence at $R_{\lambda} \approx 280$ (Run $2-1 \mathrm{~K}$ ) in figure 1 . This figure shows results at a single time. We have confirmed that the behaviors of $T$ are qualitatively independent of time, though the values of $T$ evolve in time.

First, let us look at the results shown in figure 1(a) for a low wavenumber range $\left(K \in\left[0.0020 \eta^{-1}, 0.0087 \eta^{-1}\right]\right)$, where the effect of the external force is dominant. This figure shows the energy transfer $T\left(K, K^{\prime}, t\right)$ as a function of $K^{\prime} / K$, where the energy transfers from wavenumber $K^{\prime}$ to $K$. The different curves in the plot show the results for different $K$; darker (thinner) curves correspond to larger $K$. It is clear in this figure that the Fourier modes at $K$ acquire energy from smaller wavenumber modes $\left(K^{\prime}<K\right)$ and transfer the energy to larger wavenumber modes $\left(K<K^{\prime}\right)$. These characteristics are common in the four wavenumber ranges shown in figures 1(a)-(d). However, we observe in figure 1(a) "whiskers" at the lowest wavenumber. This corresponds to the contribution from the Fourier modes which the external force $f$ directly drives. Recall that, in this turbulence, the external force is steady and therefore these modes are rather robust.

Secondly, we look at the inertial range. Figure 1(b) shows the energy transfer in the wavenumber range $K \in\left[0.0105 \eta^{-1}, 0.026 \eta^{-1}\right]$. This figure also shows that the energy transfers from lower $\left(K^{\prime}<K\right)$ to higher $K^{\prime}>K$ wavenumber modes. In contrast to the lower wavenumber range [figure 1(a)], the contribution from about the half (more precisely, $K^{\prime}=0.58 K$ ) wavenumber modes (i.e. 1.7 times larger scales) transfer the energy to $K$ than the forcing modes. Note that the peaks at $K^{\prime}=0.58 K$ is higher than the whiskers. This is also consistent with the observation in the same figure [figure 1(b)] that the energy at the wavenumber $K$ transfers most to a higher wavenumber mode at $K^{\prime}=1.7 K$ (i.e. 0.58 times smaller scales). It is further important to observe that all the curves for different $K$ collapse almost perfectly. This means that the energy transfer due to vortex stretching occurs in a self-similar manner, and that the energy is conserved by this cascading process. In other words, the viscous effect is negligible in this wavenumber range (i.e. the inertial range). We may verify the self-similarity in figure 2, where we show $K_{\max }^{\prime} / K$ and $K_{\min }^{\prime} / K$ as functions of $K$. Here, $K_{\max }^{\prime}$ and $K_{\min }^{\prime}$ denote the wavenumbers which attain the maximum and minimum of the energy transfer:

$$
K_{\max }^{\prime}(K, t):=\underset{K^{\prime}}{\operatorname{argmax}} T\left(K, K^{\prime}, t\right) \quad \text { and } \quad K_{\min }^{\prime}(K, t):=\underset{K^{\prime}}{\operatorname{argmin}} T\left(K, K^{\prime}, t\right) .
$$

We can see that $K_{\max }^{\prime} \sim K$ and $K_{\min }^{\prime} \sim K$ in the wavenumber range $K \in$ $\left[0.01 \eta^{-1}, 0.04 \eta^{-1}\right]$.

Thirdly, we look at the dissipation range. Figure 1(c) shows the energy transfer in a higher wavenumber range: $K \in\left[0.031 \eta^{-1}, 0.16 \eta^{-1}\right]$. The behaviors of the energy transfer are qualitatively different from those in the inertial range. (i) There is no collapse of the curves because the amount of energy transfer decreases with wavenumber, since the energy is dissipated due to viscosity. (ii) the valleys of the energy transfer located always at $K^{\prime}=1.7 K$, whereas the wavenumber $K^{\prime}$ giving the maximum behaves in a non-trivial manner (see figure 2p). This implies the disruption of the self-similarity, which is also reasonable because of the viscous effects. 


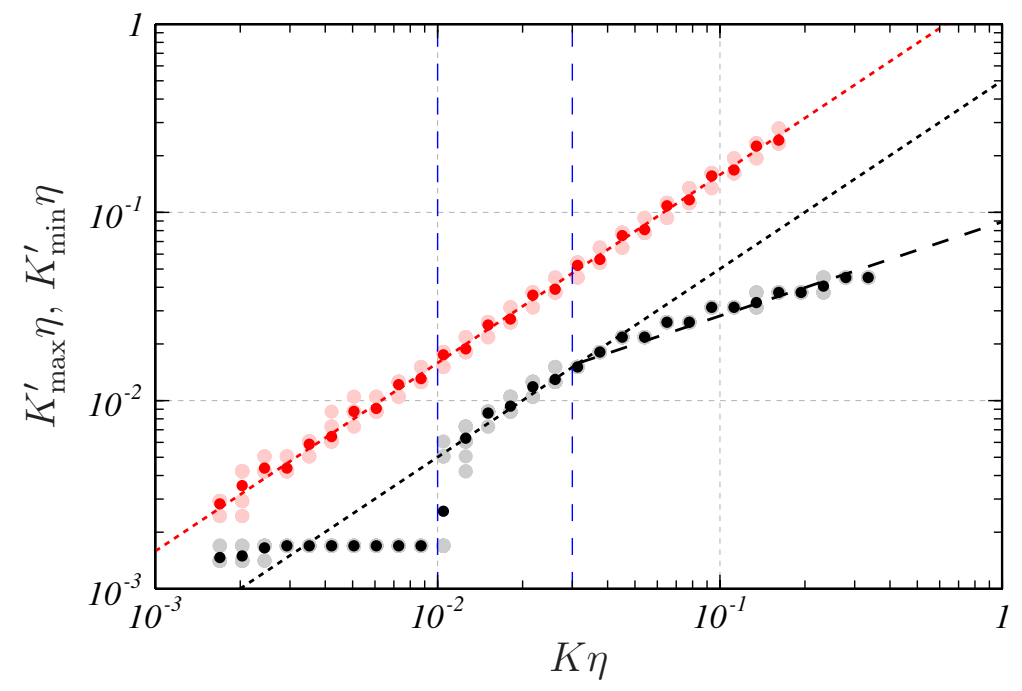

Figure 2. The wavenumbers $K_{\max }^{\prime}$ (gray symbols) and $K_{\min }^{\prime}$ (pink) giving the maximum and minimum of $T\left(K, K^{\prime}, t\right)$, respectively, as a function of $K$. We plot the data at 20 different instants. The red and black dots show the temporal averages of $K_{\max }^{\prime}$ and $K_{\min }^{\prime}$, respectively. The dotted and dashed lines indicate $K^{\prime} \sim K$ and $K^{\prime} \sim K^{1 / 2}$, respectively. The two blue vertical dashed lines indicate $K=0.01 \eta^{-1}$ and $0.03 \eta^{-1}$. Results of Run $2-1 \mathrm{~K}\left(R_{\lambda} \approx 280\right)$. We do not show $K_{\min }^{\prime}$ for $K \eta>0.19$ because the numerical resolution is not sufficient to determine it.

Fourthly, we look at the far dissipation range. Figure 1(d) shows the energy transfer in $K \in\left[0.19 \eta^{-1}, 0.33 \eta^{-1}\right]$. The behaviors of the energy transfer in this high wavenumber range is further different from those in figure 1.(c). Though the Fourier modes acquire the energy form larger scales, almost no energy transfers to higher wavenumbers.

Thus, the energy transfer behaves differently in the four wavenumber ranges. The bounds of these ranges are located at $K=0.01 \eta^{-1}, 0.03 \eta^{-1}$ and $0.19 \eta^{-1}$. These wavenumbers are shown in figure 3 , in which we plot the energy and its dissipation spectra:

$$
E(K, t):=\oint_{K \leq\left|K^{\prime}\right|<K+1}\left|\widehat{u}\left(K^{\prime}, t\right)\right|^{2} d \Omega \quad \text { and } \quad D(K, t):=\nu K^{2} E(K, t)
$$

The wavenumbers $K=0.01 \eta^{-1}$ and $0.03 \eta^{-1}$ correspond to the lower and higher ends of the $-5 / 3$ power law, respectively. We expect that the lower bound normalized by $\eta^{-1}$ depends on the Reynolds number, whereas the higher bound does not. Furthermore, the boundary $K=0.19 \eta^{-1}$ between the dissipation and far dissipation ranges is located at the peak of the energy dissipation spectrum. In summary, we can describe the scale-local energy transfer once we define it by using the enstrophy production rate as in $(2.6)$. 

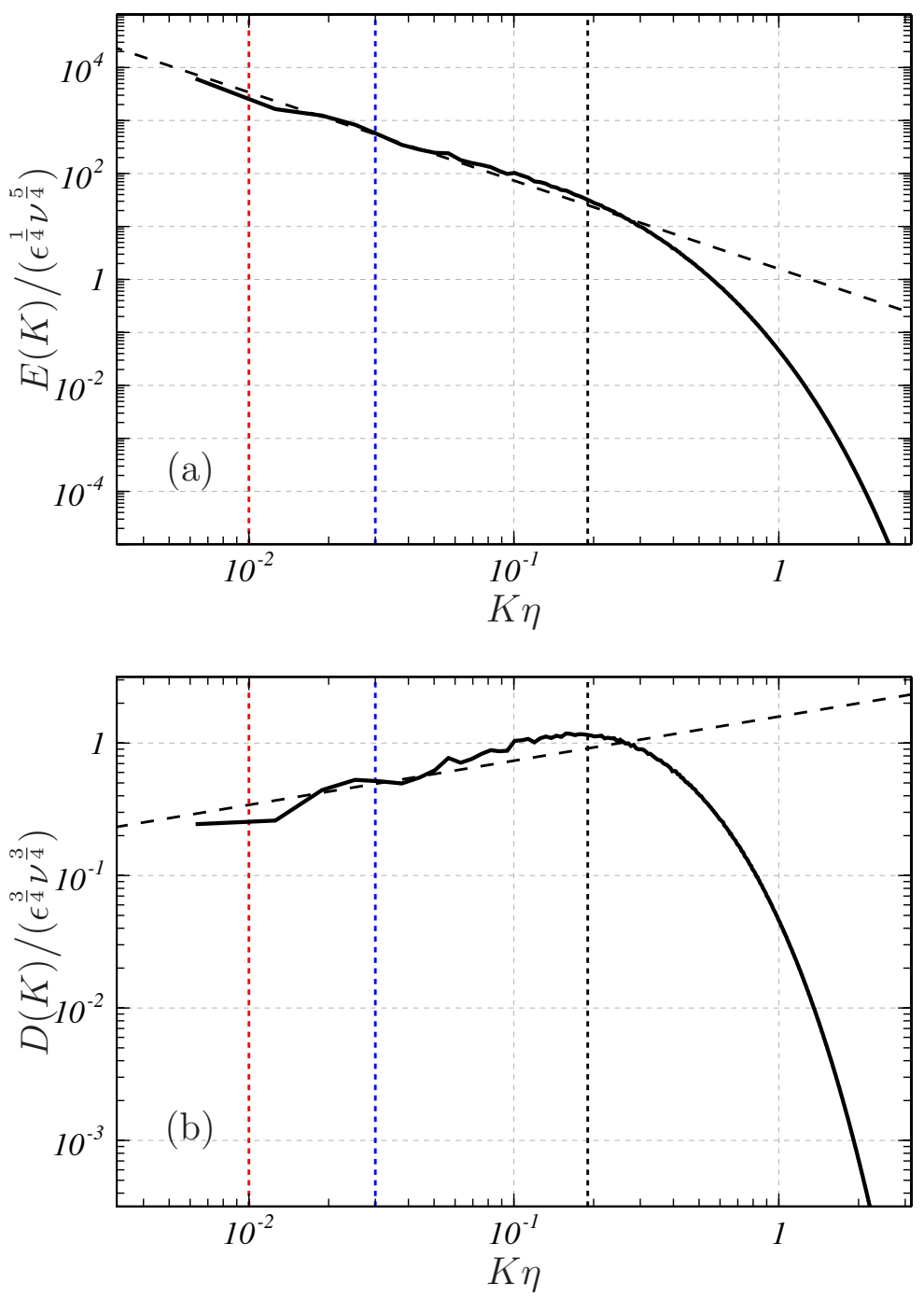

FiguRE 3. (a) Energy and (b) energy dissipation spectrum (Results of Run $2-1 \mathrm{~K} ; R_{\lambda} \approx 280$ ). Red, blue and black dotted vertical lines indicate $K=0.01 \eta^{-1}$ (the lower end of the inertial range) $0.03 \eta^{-1}$ (the lower end of the dissipation range) and $0.19 \eta^{-1}$ (the lower end of the far dissipation range). Black dashed lines indicate (a) $-5 / 3$ and (b) $1 / 3$ power laws.

2.3.2. Enstrophy production rate in the far dissipation range. The results in the previous subsection clarify the usefulness of the energy transfer $T\left(K, K^{\prime}, t\right)$ defined by the enstrophy production rate in the description of the energy cascading process. This encourages us to estimate the maximum enstrophy production rate in the far dissipation range in order to investigate the possibility of the blowup of solutions to the Navier-Stokes equation (see Sec. 3). 


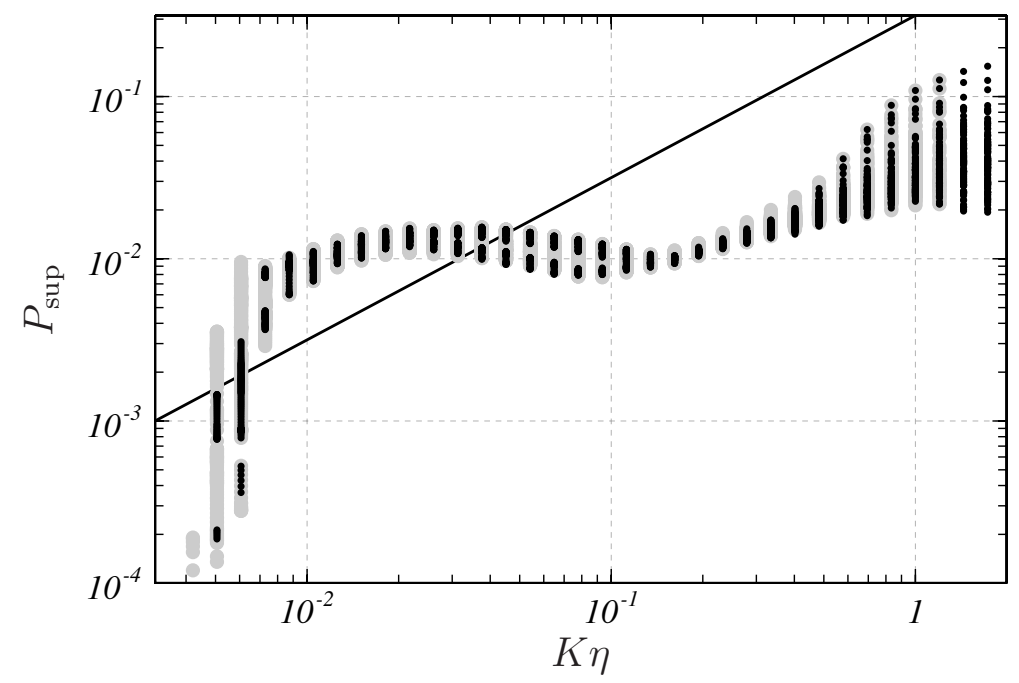

Figure 4. Maximum $P_{\text {sup }}$, defined by (2.11), of the normalized enstrophy production rate. We show the results with two different numerical resolutions with a common value of the kinematic viscosity $\nu$; gray symbols, $N^{3}=1024^{3}$, Run $8-1 \mathrm{~K}, R_{\lambda} \approx 120$; black symbols, $N^{3}=2048^{3}$, Run $8-2 \mathrm{~K}, R_{\lambda} \approx 130$. Solid black line indicates $\beta=1$.

For this purpose (i.e. to investigate the dynamics in the far dissipation range), we use the DNS results with larger $\nu$ (Runs $8-1 \mathrm{~K}$ and 8-2K). Recall that we have conducted DNS with a common value of $\nu$ but with different resolutions $N^{3}$. We plot in figure 4 the maximum

$$
P_{\text {sup }}(K, t):=\sup _{K^{\prime}} P\left(K, K^{\prime}, t\right)
$$

of the normalized enstrophy production rate

$$
P\left(K, K^{\prime}, t\right):=\frac{\int_{\mathbb{T}^{3}} \omega(K, x, t) \cdot \nabla u\left(K^{\prime}, x, t\right) \cdot \omega(K, x, t) d x}{\|\omega(K, t)\|_{L^{2}}^{2}\left\|\omega\left(K^{\prime}, t\right)\right\|_{L^{2}}} .
$$

The different symbols in the figure denote the results with different resolutions. We may confirm that the results are statistically independent of the resolution $N^{3}$, although the instantaneous values are, of course, dependent on the runs. In particular, we observe that the temporal fluctuation of $P_{\text {sup }}$ is quite large in the far dissipation range $\left(K>0.1 \eta^{-1}\right)$, but the amplitude of the fluctuations is also independent of the resolution.

The solid straight line shown in this $\log$-log plot indicates $\beta=1$ in the main theorem. The plotted function seems a concave function of $K$ in the far dissipation range, in particular, it seems to satisfy

$$
P_{\text {sup }}(K) \leq C K^{\beta}
$$

for some $\beta<1$ and $C>0$ which is independent of $K$. This result encourages us to develop the mathematics in terms of the energy transfer due to the vortex 
stretching. However, the estimation of $P_{\text {sup }}$ in the further higher dissipation range is numerically challenging because of the finite digits of the numerical accuracy, and we must leave this for a future numerical study.

\section{Global existence of Smooth flow on the NS turbulence.}

In this section, with the aid of concavity of the normalized enstrophy production rate $P_{\text {sup }}$ (defined in (2.11) in the far dissipation range, we show that the corresponding solution to the Navier-Stokes equation $(2.1)$ is smooth enough. Let us define the inhomogeneous Sobolev spaces $H^{s}$ as follows:

$$
H^{s}\left(\mathbb{T}^{3}\right):=\left\{u=\sum_{K \in \mathbb{Z}^{3}} \hat{u}(K) e^{i K \cdot x} \mid\|u\|_{H^{s}}:=\left(\sum_{K \in \mathbb{Z}^{3}}\left(1+|K|^{2}\right)^{s}|\hat{u}(K)|^{2}\right)^{1 / 2}<\infty\right\},
$$

and let us define the band-pass filter (Littlewood-Paley decomposition) as follows:

$$
\omega^{k}:=\mathcal{F}^{-1}\left[\chi_{\left[\frac{2^{k}}{\sqrt{2}}, \sqrt{2} 2^{k}\right)} \mathcal{F}[\omega]\right]
$$

where $\chi$ is the characteristic function. Then, if the vorticity $\omega$ is mean zero, we can decompose it as follows:

$$
\omega=\sum_{k \in \mathbb{Z}_{\geq-1}} \omega^{k}
$$

and we see

$$
\|\omega\|_{H^{s}} \approx\left(\sum_{j \in \mathbb{Z}_{\geq-1}} 2^{2 s j}\left\|\omega^{j}\right\|_{L^{2}}^{2}\right)^{1 / 2} .
$$

Now we decompose the vortex stretching term as follows:

$$
\begin{aligned}
\int_{\mathbb{T}^{3}}(\omega \cdot \nabla) u \cdot \omega & =\sum_{k \in \mathbb{Z}_{\geq-1}} \sum_{m \in \mathbb{Z}_{\geq-1}} \sum_{\ell \in \mathbb{Z}_{\geq-1}} \int_{\mathbb{T}^{3}}\left(\omega^{\ell} \cdot \nabla\right) u^{m} \cdot \omega^{k} \\
& =\sum_{k \in \mathbb{Z}_{\geq-1}} \sum_{m \in \mathbb{Z}_{\geq-1}} \sum_{\ell \in \mathbb{Z}_{\geq-1}} \widetilde{P}\left(2^{k}, 2^{m}, 2^{\ell}, t\right)\left\|\omega^{\ell}\right\|_{L^{2}}\left\|\omega^{m}\right\|_{L^{2}}\left\|\omega^{k}\right\|_{L^{2}},
\end{aligned}
$$

where $\widetilde{P}$ is defined as follows:

$$
\widetilde{P}\left(2^{k}, 2^{m}, 2^{\ell}, t\right):=\frac{\int_{\mathbb{T}^{3}} \omega^{\ell}(x, t) \cdot \nabla u^{m}(x, t) \cdot \omega^{k}(x, t) d x}{\left\|\omega^{\ell}(t)\right\|_{L^{2}}\left\|\omega^{m}(t)\right\|_{L^{2}}\left\|\omega^{k}(t)\right\|_{L^{2}}} .
$$

Remark 2. By DNS, we have already observed that

$$
\widetilde{P}_{\text {sup }} \sim P_{\text {sup }}
$$

where

$$
\widetilde{P}_{\text {sup }}(K, t)=\sup _{K^{\prime}, K^{\prime \prime}} \widetilde{P}\left(K, K^{\prime}, K^{\prime \prime}, t\right) .
$$

Physically, the coincidence of $P_{\text {sup }}$ and $\widetilde{P}_{\text {sup }}$ is an important issue, thus, as the sequence of this study, we will provide another research article elsewhere. 
We assume there exist $\beta<1$ and a constant $C>0$ (independent of $k$ ) such that

$$
\sup _{t \in\left[0, t_{*}\right)} \sup _{\ell, m} \widetilde{P}\left(2^{k}, 2^{m} \cdot 2^{\ell}, t\right) \leq C 2^{k \beta}
$$

as long as the solution $u(t)$ exists in $t \in\left[0, t_{*}\right)$. By the DNS result in Subsection 2.3 .2 it seems that the NS turbulence satisfies (3.3) Then we obtain the following regularity criterion.

Theorem 1. Let $f \in L^{\infty}\left(0, \infty ; H^{-1}\left(\mathbb{T}^{3}\right)\right)$ and $u_{0} \in H^{1}\left(\mathbb{T}^{3}\right)$ with $\int_{\mathbb{T}^{3}} f=\int_{\mathbb{T}^{3}} u_{0}=0$ and $\nabla \cdot f=\nabla \cdot u_{0}=0$. Suppose that $u \in C\left(\left[0, t_{*}\right): H^{1}\left(\mathbb{T}^{3}\right)\right)$ is a local-in-time strong solution to the three-dimensional Navier-Stokes equation (2.1). If $\beta<1$ for $t \in\left[0, t_{*}\right)$, then $u$ can be extended to the strong solution up to the time $t_{* *}$ with $t_{* *}>t_{*}$.

Remark 3. By applying a bootstrapping argument for regularity (see the proof of Theorem 2 in 27] for example) and another bootstrapping argument for existence time (see the proof of Theorem 4 in [30] for example), combining with the a-priori bound (3.5), we can show that the solution exists in an arbitrary time interval, and is smooth enough in both space and time, if the external force $f$ is smooth enough.

Proof. The proof is rather elementary. Our calculation is simply based on [1, Theorem 3.2] and [14, Section 3.3]. They considered global existence of solutions to the incompressible 3D Navier-Stokes equation with the Coriolis force in a periodic domain. The key point is to construct an a-priori estimate in $H^{1}$. First, we estimate (3.1). Let us choose $\delta>0$ such that $\beta+\delta<1$. By Bony's paraproduct formula (see 2] for example), combining Hölder's inequality for sums, we have

$$
\begin{aligned}
\int_{\mathbb{T}^{3}}(\omega \cdot \nabla) u \cdot \omega & \leq C \sum_{k \in \mathbb{Z}_{\geq-1}} \sum_{(\ell, m) \in D_{1}(k)} 2^{\beta k}\left\|\omega^{\ell}\right\|_{L^{2}}\left\|\omega^{m}\right\|_{L^{2}}\left\|\omega^{k}\right\|_{L^{2}} \\
& +C \sum_{k \in \mathbb{Z}_{\geq-1}} \sum_{(\ell, m) \in D_{2}(k)} 2^{\beta k}\left\|\omega^{\ell}\right\|_{L^{2}}\left\|\omega^{m}\right\|_{L^{2}}\left\|\omega^{k}\right\|_{L^{2}} \\
& +C \sum_{k \in \mathbb{Z}_{\geq-1}} \sum_{(\ell, m) \in D_{3}(k)} 2^{\beta k}\left\|\omega^{\ell}\right\|_{L^{2}}\left\|\omega^{m}\right\|_{L^{2}}\left\|\omega^{k}\right\|_{L^{2}},
\end{aligned}
$$

where (this is well-known Bony's paraproduct formula)

$$
\left\{\begin{array}{l}
D_{1}(k):=\left\{(\ell, m) \in\left(\mathbb{Z}_{\geq-1}\right)^{2}: k-1 \leq m \leq k+1, \quad \ell \leq m-1\right\}, \\
D_{2}(k):=\left\{(\ell, m) \in\left(\mathbb{Z}_{\geq-1}\right)^{2}: k-1 \leq \ell \leq k+1, \quad m \leq \ell-1\right\}, \\
D_{3}(k):=\left\{(\ell, m) \in\left(\mathbb{Z}_{\geq-1}\right)^{2}: \ell \geq k-1, \quad \ell-1 \leq m \leq \ell+1\right\} .
\end{array}\right.
$$


Then direct calculations yield

$$
\begin{aligned}
& \lesssim\|\omega\|_{L^{2}} \sum_{k \in \mathbb{Z}_{\geq-1}} 2^{\beta k}\left\|\omega^{k}\right\|_{L^{2}} \sum_{\ell \leq k}\left\|\omega^{\ell}\right\|_{L^{2}} \\
& +\|\omega\|_{L^{2}} \sum_{k \in \mathbb{Z}_{\geq-1}} 2^{\beta k}\left\|\omega^{k}\right\|_{L^{2}} \sum_{m \leq k}\left\|\omega^{m}\right\|_{L^{2}} \\
& +\sum_{k \in \mathbb{Z}_{\geq-1}} 2^{\beta k}\left\|\omega^{k}\right\|_{L^{2}} \sum_{\ell \geq k-1}\left\|\omega^{\ell}\right\|_{L^{2}}\left(\left\|\omega^{\ell-1}\right\|_{L^{2}}+\left\|\omega^{\ell}\right\|_{L^{2}}+\left\|\omega^{\ell+1}\right\|_{L^{2}}\right) \\
& \lesssim\|\omega\|_{L^{2}}^{2}\left(\sum_{k \in \mathbb{Z}_{\geq-1}} 2^{2(\beta+\delta) k}\left\|\omega^{k}\right\|_{L^{2}}^{2}\right)^{1 / 2}\left(\sum_{k \in \mathbb{Z}_{\geq-1}} 2^{-2 \delta k} k\right)^{1 / 2} \\
& +\|\omega\|_{L^{2}}^{2}\left(\sum_{k \in \mathbb{Z}_{\geq-1}} 2^{2(\beta+\delta) k}\left\|\omega^{k}\right\|_{L^{2}}^{2}\right)^{1 / 2}\left(\sum_{k \in \mathbb{Z} \geq-1} 2^{-2 \delta k}\right)^{1 / 2} \\
& \lesssim\|\omega\|_{H^{\beta+\delta}}\|\omega\|_{L^{2}}^{2} .
\end{aligned}
$$

Here we used the fact that

$$
\sum_{\ell \leq k} a_{\ell} \leq\left(\sum_{\ell \leq k} a_{\ell}^{2}\right)^{1 / 2}\left(\sum_{\ell \leq k} 1\right)^{1 / 2} \quad \text { for } \quad a_{\ell} \geq 0 .
$$

We just proceed the $H^{1}$ energy estimate as (recall $\|\omega\|_{L^{2}} \approx\|u\|_{H^{1}}$ with $\int_{\mathbb{T}^{3}} u=0$ )

$$
\frac{d}{d t}\|u(t)\|_{H^{1}}^{2}+2 \nu\|u(t)\|_{H^{2}}^{2} \lesssim\|u(t)\|_{H^{1}}^{2}\|u(t)\|_{H^{1+\beta+\delta}}+\|f\|_{H^{1}}\|u\|_{H^{1}} .
$$

By interpolation and Young's inequality (note $\beta+\delta \leq 1$ ),

$$
\begin{aligned}
& \|u\|_{H^{1}}^{2}\|u\|_{H^{1+\beta+\delta}} \lesssim\|u\|_{H^{1}}^{2}\|u\|_{H^{2}} \lesssim \nu^{-1}\|u\|_{H^{1}}^{4}+\nu\|u\|_{H^{2}}^{2}, \\
& \|f\|_{H^{1}}\|u\|_{H^{1}} \lesssim\|f\|_{H^{1}}^{2}+\|u\|_{H^{1}}^{2}
\end{aligned}
$$

and hence,

$$
\frac{d}{d t}\|u(t)\|_{H^{1}}^{2}+\nu\|u(t)\|_{H^{2}}^{2} \leq\left(1+\nu^{-1}\|u(t)\|_{H^{1}}^{2}\right)\|u(t)\|_{H^{1}}^{2}+\|f\|_{H^{1}}^{2}, \quad t>0
$$

On the other hand, we immediately have the energy inequality:

$$
\frac{d}{d t}\|u(t)\|_{L^{2}}^{2}+2 \nu\|u(t)\|_{H^{1}}^{2} \lesssim\|f(t)\|_{H^{-1}}\|u(t)\|_{H^{1}} .
$$

Thus, by the absorbing argument, we have

$$
\|u(t)\|_{L^{2}}^{2}+\nu \int_{0}^{t}\|u(t)\|_{H^{1}}^{2} \lesssim \nu^{-1} \int_{0}^{t}\|f(t)\|_{H^{-1}}^{2}+\|u(0)\|_{L^{2}}^{2}
$$


By the Gronwall inequality, we then have

$$
\begin{aligned}
& \|u(t)\|_{H^{1}}^{2} \lesssim\left\|u_{0}\right\|_{H^{1}}^{2} e^{\int_{0}^{t}\left(1+\nu^{-1}\|u(s)\|_{H^{1}}^{2}\right) d s}+\int_{0}^{t} e^{\int_{\tau}^{t}\left(1+\nu^{-1}\|u(s)\|_{H^{1}}^{2}\right) d s}\|f(\tau)\|_{H^{1}}^{2} d \tau \\
& \lesssim\left\|u_{0}\right\|_{H^{1}}^{2} \exp \left(t+\nu^{-2} \int_{0}^{t}\|f(s)\|_{H^{-1}}^{2} d s+\nu^{-1}\left\|u_{0}\right\|_{L^{2}}^{2}\right) \\
& +\exp \left(t+\nu^{-2} \int_{0}^{t}\|f(s)\|_{H^{-1}}^{2} d s+\nu^{-1}\left\|u_{0}\right\|_{L^{2}}^{2}\right) \int_{0}^{t}\|f(\tau)\|_{H^{1}}^{2} d \tau<\infty
\end{aligned}
$$

for $t \in\left[0, t_{*}\right)$. By this a-priori estimate combining local existence result (see [14, Theorem 1.2] for example), we can immediately prove the main theorem.

\section{Reformulation of the Kolmogorov-Richardson EnERgy CAsCAde}

In this section, with the aid of the DNS result in Sec. 2, we describe the Kolmogorov-Richardson energy cascade in terms of vortex stretching. In what follows (including the next section), we employ $\mathbb{R}^{3}$ which is as an approximation domain of $\mathbb{T}^{3}$. Also we simply write $\int f:=\int_{\mathbb{R}^{3}} f(x) d x$. Let $u$ be a smooth solution to the 3D-Navier-Stokes equation (2.1) (but replace $\mathbb{T}^{3}$ to $\mathbb{R}^{3}$ ), and $\omega$ be the corresponding vorticity. Let

$$
\bar{\omega}_{K}(t, x):=\mathcal{F}_{\xi}^{-1}\left[\chi_{A_{K}}(\xi) \hat{\omega}(t, \xi)\right](x),
$$

where (coarse-graining in an annulus, Littlewood-Paley decomposition)

$$
A_{K}:=\left\{\xi \in \mathbb{R}^{3}: K / \sqrt{\alpha} \leq|\xi| \leq \sqrt{\alpha} K\right\}
$$

and $\alpha>1$ is a prescribed constant expressing the ratio of adjacent scales (see Figure 1). In what follows we take wavenumber $K$ from $\alpha^{\mathbb{Z}}$. Also, in the following arguments, we assume that the spatially integrated value of any quantity is independent of time because the size of the domain is much larger than the correlation length (the integral length) of the flow, that is,

$$
\frac{d}{d t}\left\|\bar{\omega}_{K}(t)\right\|_{L^{2}}^{2}=0 \quad \text { for any } \quad K \quad \text { and } \quad t \geq 0 .
$$

Our specific purpose in this section is to approximate the following vortex stretching term:

$$
\int(\omega \cdot \nabla) u \cdot \bar{\omega}_{K} .
$$

Note that $\omega=\sum_{K \in \alpha^{\mathbb{Z}}} \bar{\omega}_{K}$ and $u=\sum_{K \in \alpha^{\mathbb{Z}}} \bar{u}_{K}$. First, we propose a new hypothesis. For the original version of Kolmogorov hypothesis, see [15], for a recasted version, see [6, Section 6].

Outline of our hypothesis. The crucial point of our hypothesis is that there exists a universal vortex stretching/compressing mechanism, independent of the energy input rate $\epsilon$ (which will appear later). More precisely, a pair of tubular vortices $W_{j, K}$ are stretching several tubular vortices in the adjacent smaller scale, or, several tubular vortices are compressing a pair of tubular vortices in the adjacent larger scale. Also, supports of these tubular vortices (with compact supports) are disjoint. We conjecture that such vortex stretching/compressing in adjacent two scales are the dominant event, so, in our cascade picture, we exclude multiscale 
events. Note that, by Remark 6, due to the uncertainty principle for the Fourier transform, we expect that the three-wave interaction of this adjacent scale event is rather nonlocal (c.f. [19]).

Balance of vortex stretching/compressing. Vortex stretching and compressing are balanced if (4.2) holds. See also (5.3).

A pair of tubular vortices. Let $W_{j, K} \in C_{c}^{\infty}\left(\mathbb{R}^{3}\right)$ represents a pair of "normalized" tubular vortices, where $j$ and $K$ are important indexes, will appear later. First we reasonably assume

$$
\operatorname{supp} W_{j, K} \cap \operatorname{supp} W_{j^{\prime}, K}=\emptyset \quad \text { for } \quad j \neq j^{\prime} .
$$

Next we define $\widetilde{W}_{j, K}(x)$ as follows:

$$
\widetilde{W}_{j, K}(x):=\mathcal{F}^{-1}\left[\chi \mathcal{F}\left[W_{j, K}\right]\right](x),
$$

where $\chi$ is a characteristic function such that

$$
\chi(\xi)= \begin{cases}1 & \frac{1}{\sqrt{\alpha}} \leq|\xi| \leq \sqrt{\alpha} \\ 0 & \text { otherwise }\end{cases}
$$

Note that $\operatorname{supp} \mathcal{F}\left[\widetilde{W}_{j, K}\right] \subset A_{1}$ and $\widetilde{W}_{j, K}$ is decaying (not compactly supported) in the physical space, due to the uncertainty principle. We assume that

$$
\int_{\mathbb{R}^{3}}\left|W_{j, K}(x)\right|^{2} d x=\gamma
$$

for some prescribed (non-dimensional) constant $\gamma$ which is independent of all parameters.

Remark 4. In the real turbulence, this $\gamma$ is rather probability distribution.

By Parseval's identity, we see that

$$
\int_{\mathbb{R}^{3}}\left|\widetilde{W}_{j, K}(x)\right|^{2} d x=\int_{\mathbb{R}^{3}}\left|W_{j, K}(x)\right|^{2} d x+\int_{\mathbb{R}^{3}}\left|\widetilde{W}_{j, K}(x)-W_{j, K}(x)\right|^{2} d x .
$$

Also assume these $\widetilde{W}_{j, K}$ and $W_{j, K}$ are close to each other, that is,

$$
\frac{\int_{\mathbb{R}^{3}}\left|W_{j, K}(x)-\widetilde{W}_{j, K}(x)\right|^{2} d x}{\int_{\mathbb{R}^{3}}\left|W_{j, K}(x)\right|^{2} d x} \leq \delta<1
$$

for some small $\delta>0$. In Appendix, we give a typical example of tubular vortices $W_{j, K}$, and we estimate $\delta$ by using it.

Decomposition of vorticity field. In the inertial range, we employ the following hypothesis. For any fixed $t$, assume that $\bar{\omega}_{K}$ is mainly expressed by $\left\{W_{j, K}\right\}_{j}$, and is independent of $\nu$ and $\delta$, as follows:

$$
\bar{\omega}_{K}(t, x)=g K^{-H+1}\left(\sum_{j=1}^{c K^{D}} W_{j, K}(K x)\right),
$$

where $c, g, H, D \in \mathbb{R}_{\geq 0}$. Rigorously, there must exist a small perturbation (in order to recover divergence-free and compact support in the Fourier space) depending on 
$\nu$ and $\delta$, but we neglect it. $H$ and $D$ express the Hölder exponent and the fractal dimension respectively. Rigorously, we need to require $c K^{D}$ to be integer. In this case we slightly approximate $c$ and $D$. This $D$ is ideally determined by physical experiments. For example, in [24, we have investigated this exponent by numerical computations. In particular, we identified the tubular vortices in each scales by the low-pressure method, and we figured out that the hierarchy of stronger tubular vortices is indeed intermittent with the dimension $D$ smaller than 3 . The above assumptions are supported by 24] in some extent.

Remark 5. (The specific aim of this paper.) The vorticity version of the LittlewoodPaley spectra $E_{L P}(K)$ is given by

$$
E_{L P}(K):=K^{-3}\left\|\bar{\omega}_{K}\right\|_{L^{2}}^{2}=c \gamma g^{2} K^{-\frac{5}{3}} K^{-\frac{1}{3}(3-D)} K^{\frac{2}{3}(-3 H-2+D)}
$$

(c.f. Sec. 2 in 3 for the definition of the Littlewood-Paley spectra). Thus, our specific aim is to figure out the constants $g$ and $H$. Note that this $E_{L P}$ dimensionally coincides with another $E$ in $(2.10)$.

The external force. For fixed $c>0$, we assume that, for any energy input rate $\epsilon$ and wavenumber $K_{f}$, we can construct an external force $f$ such that $\operatorname{supp} \hat{f} \subset A_{K_{f}}$ and

$$
\frac{1}{K_{f}^{2}} \int \overline{(\nabla \times f)}_{K_{f}} \cdot \omega=\epsilon,
$$

where $\omega$ is a solution to the Navier-Stokes (vorticity) equation.

Hierarchy of tubular vortices. Let $K_{ \pm}=\alpha^{\mp 1} K$ and let us assume that a pair of tubular vortices $W_{J, K_{+}}$stretches (through Biot-Savart law) $W_{j, K}\left(j \in{ }^{\exists} \Omega_{J, K}^{+} \subset\right.$ $\left.\left\{1,2, \cdots, c K^{D}\right\}\right)$ and assume disjointness $\Omega_{J, K}^{+} \cap \Omega_{J^{\prime}, K}^{+}=\emptyset\left(J \neq J^{\prime}\right)$. At the same time, this $W_{j, K}$ compresses $W_{J, K_{+}}$. If $W_{J, K_{+}}$does not stretch anything, then we regard $\Omega_{J, K}^{+}$as an empty set. In the forcing scale, the external force $\nabla \times f$ stretches $W_{j, K_{f}}\left(j \in \Theta^{\exists} \Omega_{K_{f}}^{+} \subset\left\{1,2, \cdots, c\left(K_{f}\right)^{D}\right\}\right)$. Let

$$
\Omega_{K}^{+}=\bigcup_{J=1}^{c K_{+}^{D}} \Omega_{J, K}^{+} .
$$

To the contrary, assume that, for any $j \in\left(\Omega_{K}^{+}\right)^{c}:=\left\{1,2, \cdots, c K^{D}\right\} \backslash \Omega_{K}^{+}, W_{j, K}$ is compressed by $W_{j^{\prime}, K_{-}}\left(j^{\prime} \in{ }^{\exists} \Omega_{j, K}^{-} \subset \mathbb{N}\right)$ through Biot-Savart law. At the same time, $W_{j^{\prime}, K_{-}}$is stretched by this $W_{j, K}$. Note that $\Omega_{J, K}^{+}=\Omega_{J, K_{+}}^{-}$and $\left|\Omega_{K}^{+}\right|+\left|\left(\Omega_{K}^{+}\right)^{c}\right|=$ $K^{D}$. The construction of this scenario is inspired by Movie S9 in "Supplementary materials" in [16]. In this movie, we can observe forming an ordered array of counter-rotating secondary vortex filaments perpendicular to the primary cores. We can also observe secondary filaments interacting to form a new generation of perpendicular tertiary vortex filaments.

Clarification of $\alpha$ and $\left|\Omega_{j, K}^{+}\right|$. We crucially assume $\alpha$ and $\left|\Omega_{j, K}^{+}\right|\left(=\left|\Omega_{j, K}^{-}\right|\right)$are non-dimensional prescribed constants (ideally determined by physical experiments, see also Figure 11, that is, independent of $K$ and $j$. Rigorously this $\left|\Omega_{j, K}^{+}\right|$is integer, but here we are implicitly spatially averaging it, to be a positive real number. We conjecture that this $\left|\Omega_{j, K}^{+}\right|$is determined by an unstable mode (which is independent of any type of disturbance) induced by the elliptical instability (see Subsection 2.2 
in [13, see also [16]). More precisely, the elliptical instability originates from the parametric excitation of Kelvin modes in the vortex cores in each tubular vortices $W_{j, K}$.

Ratios of the number of tubular vortices: being stretched and being compressed. Let $\tau_{+}(K)$ and $\tau_{-}(K)$ be ratios of the number of tubular vortices, being stretched and being compressed, respectively. Since in the statistically steady state, these ratios could be estimated by the ratio of turnover time in adjacent scales, it follows that

$$
\text { turnover time } \sim \frac{\text { length }}{\text { velocity }} \sim g^{-1} K^{H-1} .
$$

Thus we can define $\tau_{ \pm}$(satisfying $\tau_{+}+\tau_{-}=1$ ) as follows:

$$
\begin{aligned}
& \tau_{+}(K):=\frac{K^{H-1}}{K^{H-1}+K_{-}^{H-1}}=\frac{1}{1+\alpha^{H-1}}, \\
& \tau_{-}(K):=\frac{K_{-}^{H-1}}{K^{H-1}+K_{-}^{H-1}}=\frac{1}{\alpha^{-H+1}+1} .
\end{aligned}
$$

Consequently $\tau_{ \pm}$are independent of $K$.

Vortex stretching/compressing relation vs spatial and statistical independence. For tubular vortices $W_{j_{1}, K_{1}}$ and $W_{j_{2}, K_{2}}$, let us define vortex stretching/compressing relations as follows:

- Vortex stretching relation:

$$
V^{+}:=\left\{\left(j_{1}, K_{1}, j_{2}, K_{2}\right): j_{1} \in \Omega_{j_{2}, K_{1}}^{+} \text {and } K_{2}=\alpha^{-1} K_{1}\right\}
$$

- Vortex compressing relation:

$$
V^{-}:=\left\{\left(j_{1}, K_{1}, j_{2}, K_{2}\right): j_{2} \in \Omega_{j_{1}, K_{1}}^{-} \text {and } K_{2}=\alpha K_{1}\right\}
$$

Definition 1. (Vortex stretching/compressing relation: disjointness) We assume the following: If $W_{j_{1}, K_{1}}$ and $W_{j_{2}, K_{2}}$ have vortex stretching/compressing relation, that is, if $\left(j_{1}, K_{1}, j_{2}, K_{2}\right) \in V^{+} \cup V^{-}$, then

$$
\operatorname{supp} W_{j_{1}, K_{1}} \cap \operatorname{supp} W_{j_{2}, K_{2}}=\emptyset .
$$

Definition 2. (Spatial and statistical independence.) Let $U_{j, K}:=-\nabla \Delta^{-1} \times$ $W_{j, K}$. Then we significantly assume the following:

$$
\sum_{\left(j_{1}, K_{1}, j_{2}, K_{2}\right) \in\left(V^{+}\right)^{c} \cap\left(V^{-}\right)^{c}} \int\left(W_{j_{1}, K_{1}} \cdot \nabla\right) U_{j_{2}, K_{2}} \cdot W_{j^{*}, K^{*}}=0
$$

and

$$
\sum_{\substack{\left(j_{1}, K_{1}, j_{2}, K_{2}\right) \in V^{+} \cup V^{-},\left(j^{*}, K^{*}\right) \\\left(j_{1}, K_{1}\right) \neq\left(j^{*}, K^{*}\right) \text { and }\left(j_{2}, K_{2}\right) \neq\left(j^{*}, K^{*}\right)}} \int\left(W_{j_{1}, K_{1}} \cdot \nabla\right) U_{j_{2}, K_{2}} \cdot W_{j^{*}, K^{*}}=0 .
$$

for any fixed $j^{*}$ and $K^{*}$.

By Definitions 1 and 2, we have that

$$
\int(\omega \cdot \nabla) u \cdot \bar{\omega}_{K}=\int\left(\bar{\omega}_{K} \cdot \nabla\right) \bar{u}_{K_{+}} \cdot \bar{\omega}_{K}+\int\left(\bar{\omega}_{K} \cdot \nabla\right) \bar{u}_{K_{-}} \cdot \bar{\omega}_{K}
$$




\section{Normalization of vortex stretching/compressing.}

Let $\omega_{K}^{*}(x):=\bar{\omega}_{K}(x) /\left(g K^{-H+1}\right)$ and let $\omega_{K}^{* *}(x):=\omega_{K}^{*}\left(K^{-1} x\right)$. Then we see that

$$
\begin{aligned}
& \int\left(\bar{\omega}_{K} \cdot \nabla\right) \bar{u}_{K_{+}} \cdot \bar{\omega}_{K}+\int\left(\bar{\omega}_{K} \cdot \nabla\right) \bar{u}_{K_{-}} \cdot \bar{\omega}_{K} \\
= & g^{3} K^{-3(H-1)}\left(\alpha_{+}^{-H+1} \int\left(\omega_{K}^{*} \cdot \nabla\right) u_{K_{+}}^{*} \cdot \omega_{K}^{*}+\alpha_{-}^{-H+1} \int\left(\omega_{K}^{*} \cdot \nabla\right) \cdot u_{K_{-}}^{*} \cdot \omega_{K}^{*}\right) \\
= & g^{3} K^{-3(H-1)} K^{-3}\left(\alpha_{+}^{-H+1} \int\left(\omega_{K}^{* *} \cdot \nabla\right) u_{K_{+}}^{* *} \cdot \omega_{K}^{* *}+\alpha_{-}^{-H+1} \int\left(\omega_{K}^{* *} \cdot \nabla\right) \cdot u_{K_{-}}^{* *} \cdot \omega_{K}^{* *}\right) .
\end{aligned}
$$

Note that these $\omega_{K}^{* *}, u_{K_{+}}^{* *}$ and $u_{K_{-}}^{* *}$ are precisely expressed as

$$
\begin{aligned}
\omega_{K}^{* *}(t, x) & =\sum_{j=1}^{c K^{D}} W_{j, K}(x) \\
u_{K_{+}}^{* *}(t, x)= & \sum_{j=1}^{c K_{+}^{D}} U_{j, K_{+}}\left(\alpha^{-1} x\right)=: \sum U_{j, K_{+}}, \\
u_{K_{-}}^{* *}(t, x)= & \sum_{j=1}^{c K_{-}^{D}} U_{j, K_{-}}(\alpha x)=: \sum U_{j, K_{-},}
\end{aligned}
$$

for each fixed $t$. By (4.3), then we see that

$$
\begin{aligned}
& \int\left(\omega_{K}^{* *} \cdot \nabla\right) u_{K_{+}}^{* *} \cdot \omega_{K}^{* *}=\sum_{J} \sum_{j \in \Omega_{J, K}^{+}} \int\left(W_{j, K} \cdot \nabla\right) U_{J, K_{+}} \cdot W_{j, K}, \\
& \int\left(\omega_{K}^{* *} \cdot \nabla\right) u_{K_{-}}^{* *} \cdot \omega_{K}^{* *}=\sum_{j \in\left(\Omega_{K}^{+}\right)^{c}} \sum_{j^{\prime} \in \Omega_{j, K}^{-}} \int\left(W_{j, K} \cdot \nabla\right) U_{j^{\prime}, K_{-}} \cdot W_{j, K},
\end{aligned}
$$

where

$$
\nabla U_{J, K}:=-\nabla\left(\nabla \Delta^{-1} \times W_{J, K}\right) .
$$

Let $S_{ \pm}$and $P_{ \pm}$be the strain and rotation tensors of $\nabla u_{K_{ \pm}}^{* *}$ respectively. Then we can rewrite the following vortex stretching/compressing terms:

$$
\int\left(\omega_{K}^{* *} \cdot \nabla\right) u_{K}^{* *} \cdot \omega_{K}^{* *}=\int\left(\omega_{K}^{* *}\right)^{T}\left(P_{ \pm}+S_{ \pm}\right) \cdot \omega_{K}^{* *}=\int\left(\omega_{K}^{* *}\right)^{T} S_{ \pm} \cdot \omega_{K}^{* *},
$$

where $\left(\omega_{K}^{* *}\right)^{T}$ is the row vector of $\omega_{K}^{* *}$. By the direct calculation, the rotation tensor part disappears. Let $S_{J, K_{ \pm}}$be the rate-of-strain tensor of $\nabla U_{J, K_{ \pm}}$.

Clarification of a universality of vortex stretching/compressing. We now clarify the universal vortex stretching/compressing hypothesis. In the vortex stretching case, we assume

$$
\Lambda_{+}:=\int\left(W_{j, K}\right)^{T} S_{J, K_{+}} \cdot W_{j, K}>0 \quad \text { for } \quad j \in \Omega_{J, K}^{+},
$$

where $\Lambda_{+}$is the absolute constant, in particular, independent of $\epsilon$. On the other hand, in the vortex compressing case, we assume

$$
-\Lambda_{-}:=\int\left(W_{j, K}\right)^{T} \sum_{j^{\prime} \in \Omega_{j, K}^{-}} S_{j^{\prime}, K_{-}} \cdot W_{j, K}<0 \quad \text { for } \quad j \in\left(\Omega_{K}^{+}\right)^{c},
$$


where $\Lambda_{-}>0$ is the absolute constant, in particular, independent of $\epsilon$.

Remark 6. Due to the uncertainty principle, vortex stretching in the adjacent scale

$$
\mathcal{F}\left[\sum_{j \in \Omega_{J, K}^{+}}\left(W_{j, K}\right)^{T} S_{J, K_{+}} \cdot W_{j, K}\right]
$$

has non-compact support in the Fourier space (by the same reason, the vortex compressing also has non-compact support). More precisely we expect that the following three wave interaction is nonzero:

$$
\mathcal{F}\left[\sum_{j \in \Omega_{J, K}^{+}}\left(\mathcal{F}^{-1}\left[\mathcal{F}\left[W_{j, K}\right] \chi_{A_{K_{1}}}\right]\right)^{T} \mathcal{F}^{-1}\left[\mathcal{F}\left[S_{J, K_{+}}\right] \chi_{A_{K_{2}}}\right] \cdot \mathcal{F}^{-1}\left[\mathcal{F}\left[W_{j, K}\right] \chi_{A_{K_{3}}}\right]\right]
$$

for the following nonlocal three wave combinations (c.f. (3.4)]:

$$
\left\{\begin{array}{l}
K_{1} \approx K_{3}, \quad K_{2} \ll K_{1}, \\
K_{2} \approx K_{3}, \quad K_{1} \ll K_{2}, \\
K_{1} \approx K_{2}, \quad K_{3} \ll K_{1} .
\end{array}\right.
$$

Summarizing the above arguments, we have

$$
\int\left(\omega_{K}^{* *}\right)^{T} S_{K_{ \pm}} \cdot \omega_{K}^{* *}= \pm c K^{D} \Lambda_{ \pm} \tau_{ \pm} \alpha^{\mp(-H+1)}
$$

Remark 7 . We can apply the same calculation to the convection term, and clearly it becomes zero:

$$
\int(u \cdot \nabla) \omega \cdot \bar{\omega}_{K}=\int(u \cdot \nabla) \bar{\omega}_{K} \cdot \bar{\omega}_{K}=0
$$

due to skew-symmetry.

We now summarize the important definitions of "coherent vortices" as the following:

Coherent vortices: Let us define the approximate parameters:

$$
\begin{aligned}
& C_{+}:=c \Lambda_{+} \tau_{+} \alpha^{-(-H+1)}, \\
& C_{-}:=c \Lambda_{-} \tau_{-} \alpha^{-H+1} .
\end{aligned}
$$

Note that the constants $c, \Lambda_{ \pm}$and $\alpha$ are independent of $\epsilon$.

- Inertial range (c.f. enstrophy production rate (2.6)

$$
\frac{1}{K^{2}} \int(\omega \cdot \nabla) u \cdot \bar{\omega}_{K}=g^{3}\left(C_{+}-C_{-}\right) K^{-3 H-2+D}
$$

for $K_{F}<K<{ }^{\exists} K_{\text {dis }}$. Also, from the physical observation 1.1, we mathematically assume $K_{\text {dis }} \rightarrow \infty$ as $\nu \rightarrow 0$.

- Driving force scale (beginning of the coherent vorticity)

In the forcing scale, we assume that the effect of stretching is negligible:

$$
\int\left(\bar{\omega}_{K_{f}} \cdot \nabla\right) \bar{u}_{\left(K_{f} / \alpha\right)} \bar{\omega}_{K_{f}}=0 .
$$


In other words, initially, driving force $f$ creates the largest scale vortices. Thus

$$
\frac{1}{K_{f}^{2}} \int(\omega \cdot \nabla) u \cdot \bar{\omega}_{K_{f}}=-g^{3} C_{-} K_{f}^{-3 H-2+D} .
$$

\section{Derivation of Kolmogorov's $-5 / 3$ LaW.}

In this section, we derive Kolmogorov's $-5 / 3$ law. The main theorem in this section is the following.

Theorem 5.1. Assume (4.2) and "coherent vortices". Taking $\nu \rightarrow 0$, then we have Kolmogorov's $-5 / 3$ law:

$$
E_{L P}(K):=K^{-3}\left\|\bar{\omega}_{K}\right\|_{L^{2}}^{2}=c \gamma \epsilon^{2 / 3} K^{-5 / 3} K^{-\frac{1}{3}(3-D)}
$$

for $K \geq K_{f}$.

Proof. We multiply by $\bar{\omega}_{K}$ to (2.1) integrate on both sides, divided by $K^{2}$, to obtain

$$
\begin{aligned}
& \frac{1}{2 K^{2}} \frac{d}{d t} \int_{\mathbb{R}^{3}}\left|\bar{\omega}_{K}\right|^{2}+\frac{\nu}{K^{2}} \int_{\mathbb{R}^{3}}\left|\nabla \bar{\omega}_{K}\right|^{2} \\
& +\frac{1}{K^{2}} \int_{\mathbb{R}^{3}}(u \cdot \nabla) \omega \cdot \bar{\omega}_{K}-\frac{1}{K^{2}} \int_{\mathbb{R}^{3}}(\omega \cdot \nabla) u \cdot \bar{\omega}_{K} \\
& =\frac{1}{K^{2}} \int_{\mathbb{R}^{3}}(\nabla \times f) \cdot \bar{\omega}_{K}
\end{aligned}
$$

for $K \geq K_{f}$. By the assumption: coherent vortices and (4.2), we have

$$
\frac{\nu}{K^{2}} \int_{\mathbb{R}^{3}}\left|\nabla \bar{\omega}_{K}(\cdot, x)\right|^{2} d x-g^{3}\left(C_{+}-C_{-}\right) K^{-3 H-2+D}=0
$$

for $K_{f}<K<K_{d i s}$ and

$$
\frac{\nu}{K_{f}^{2}} \int_{\mathbb{R}^{3}}\left|\nabla \bar{\omega}_{K_{f}}(\cdot, x)\right|^{2} d x+g^{3} C_{-} K_{f}^{-3 H-2+D}=\epsilon .
$$

On the other hand,

$$
\frac{\nu}{K^{2}}\left\|\nabla \bar{\omega}_{K}(\cdot)\right\|_{L^{2}}^{2} \approx \nu\left\|\bar{\omega}_{K}(\cdot)\right\|_{L^{2}}^{2}=\nu c \gamma g^{2} K^{-2 H-1+D} .
$$

Thus

$$
\left|C_{+}-C_{-}\right| \lesssim \nu c \gamma g^{-1} K^{H+1}
$$

for $K_{f}<K<K_{d i s}$ and

$$
\left|\epsilon-C_{-} g^{3} K_{f}^{-3 H-2+D}\right|<\nu c \gamma g^{2} K_{f}^{-2 H-1+D} .
$$

Since $C_{ \pm}, M$ and $H$ are independent of $\nu$ and $K$, we have

$$
C_{+}=C_{-} \quad \text { and } \quad \epsilon=C_{-} g^{3} K_{f}^{-3 H-2+D}
$$

for taking $\nu$ to zero. Then the rest argument is rather obvious. First, assume $H \neq(-2+D) / 3$, then it contradicts (5.3) if we take $K_{f}$ sufficiently large. Thus, we have $H=(-2+D) / 3$. Consequently, $H$ (and also $\tau_{ \pm}$) is independent of $\epsilon$. Second, assume $g \neq \epsilon^{1 / 3}$, then it contradicts (5.3) if we take $\epsilon$ sufficiently large. 
Thus, $g=\epsilon^{1 / 3}$. Consequently, $C_{-}=C_{+}=1$ and then $\Lambda_{ \pm}$are also determined. Therefore the Littlewood-Paley spectra $E_{L P}(K)$ is given by

$$
E_{L P}(K):=K^{-3}\left\|\bar{\omega}_{K}\right\|_{L^{2}}^{2}=c \gamma \epsilon^{2 / 3} K^{-5 / 3} K^{-\frac{1}{3}(3-D)} .
$$

This is Kolmogorov's $-5 / 3$ power law.

\section{Conclusion}

We investigated the hierarchy of coherent vortices observed in the DNS of forced turbulence in a periodic domain. First, we proved that if the normalized enstrophy production rate $P_{\text {sup }}$ is concave in the far dissipation range (more precisely, $\beta<1$ ), the corresponding solution to the Navier-Stokes equation is smooth enough. Since the DNS results shown in figure 4 seem to support the new regularity criterion that $P_{\text {sup }}$ is concave in the far dissipation range, we may conclude that the turbulence driven by the deterministic force in a periodic domain is smooth enough. Second, we construct the hierarchy of coherent vortices which is statistically self-similar in the inertial range so that we can estimate the energy transfer rate in adjacent scales due to the vortex stretching/compressing process. The crucial assumption, which is clearly supported by DNS results (figure 2), of this construction is that the process is self-similar and local in scale. Then, under this assumption, we may derive Kolmogorov's $-5 / 3$ power law of the energy spectrum of statistically stationary turbulence. We emphasize that our derivation does not require Kolmogorov's hypotheses, but instead it requires a universal mechanism of the vortex stretching and compressing. Though the present study lacks the discussion on the origin of this universality, the recent study by McKeown et al. [16] may provide with it; they investigated how the elliptical instability led to the persistence of the turbulent energy cascade through the local interactions of vortices over a hierarchy of scales.

\section{APPENDiX: A TYPICAL EXAMPLE OF ANTIPARALLEL TUBULAR VORTICES}

In this section we give a typical example of a pair of antiparallel tubular vortices, and estimate $\delta$ in (4.4), Using Fourier transform of a special function (see [21, 26, 28, 29]) with translation, we can construct a typical example of tubular vorticity $\widetilde{W}^{n, \zeta}$ within a band-pass filter:

$$
\widetilde{W}^{n, \zeta}(x):=\left(0,0, \prod_{k=1}^{n}\left(\frac{\sin \left(2^{-k} x_{1}\right)}{2^{-k} x_{1}} \frac{\sin \left(2^{-k} x_{2}\right)}{2^{-k} x_{2}} \frac{\sin \left(2^{-k} x_{3}\right)}{2^{-k} x_{3}}\right) \sin \left(\zeta x_{1}\right)\right)
$$

for some $\zeta>m:=\sum_{k=1}^{n} 2^{-k}(1 / 2 \leq m<1)$. We will fix these parameters $n \in \mathbb{Z}_{\geq 1}$ and $\zeta \in \mathbb{R}_{>0}$ later. Then we see that $\mathcal{F}\left[\widetilde{W}^{n, \zeta}\right]$ is smooth $\left(\mathcal{F}\left[\widetilde{W}^{n, \zeta}\right] \in C^{n-2}\left(\mathbb{R}^{3}\right)\right)$ and compactly supported. More precisely,

$$
\begin{aligned}
& \operatorname{supp} \mathcal{F}\left[W^{n, \zeta}\right] \subset \\
& \left\{\xi \in \mathbb{R}^{3}: \sqrt{\frac{\zeta-m}{\zeta+m}} K<\min \left\{\left|\xi_{1}\right|,\left|\xi_{2}\right|,\left|\xi_{3}\right|\right\} \text { and } \max \left\{\left|\xi_{1}\right|,\left|\xi_{2}\right|,\left|\xi_{3}\right|\right\} \leq \sqrt{\frac{\zeta+m}{\zeta-m}} K\right\}
\end{aligned}
$$

for $K=\sqrt{\frac{\zeta+m}{\zeta-m}}(\zeta-m)$ (c.f. (2.7) and (4.1). Then we can define a typical example of antiparallel tubular vortices $W^{n, \zeta}$ as follows:

$$
W^{n, \zeta}(x):=\widetilde{W}^{n, \zeta}(x) \chi_{\left[-\frac{\pi}{\zeta}, \frac{\pi}{\zeta}\right) \times[-2 \pi, 2 \pi)^{2}}\left(x_{1}, x_{2}, x_{3}\right) .
$$


In this calculable setting, we cut off in the real space (not in the Fourier space). So it is rather natural to estimate

$$
\frac{\int\left|W^{n, \zeta}-\widetilde{W}^{n, \zeta}\right|^{2}}{\int\left|\widetilde{W}^{n, \zeta}\right|^{2}} \text { instead of } \frac{\int\left|W^{n, \zeta}-\widetilde{W}^{n, \zeta}\right|^{2}}{\int\left|W^{n, \zeta}\right|^{2}} .
$$

In this paper, for the sake of simplicity, we have used the characteristic function type of band-pass filter. But, rigorously, using the overlapping smoothed LittlewoodPaley decomposition may be more effective to capture antiparallel tubular vortices. Thus it may be better to choose $\zeta$ and $m$ satisfying $\sqrt{(\zeta+m) /(\zeta-m)}>\sqrt{1.7}$. In this appendix, we examine the case $n=2$ and $\zeta=5 / 4$ (in this case $m=3 / 4$ and $\sqrt{(\zeta+m) /(\zeta-m)}=2)$. With the aid of the numerical computation, we can estimate as the following:

$$
\frac{\int\left|W^{n, \zeta}-\widetilde{W}^{n, \zeta}\right|^{2}}{\int\left|\widetilde{W}^{n, \zeta}\right|^{2}} \simeq 1-0.80 \times(0.994)^{2} \simeq 0.21
$$

From this estimate, we can naturally imagine that there exist various antiparallel tubular vortices for small $\delta$.

Acknowledgments. Research of TY was partly supported by the JSPS Grantsin-Aid for Scientific Research 17H02860, 18H01136, 18H01135 and 20H01819. SG was partly supported by the JSPS Grants-in-Aid for Scientific Research 20H02068

and 20K20973. This work was done while TY was an associate professor at the University of Tokyo, Japan.

\section{REFERENCES}

1. A. Babin, A. Mahalov and B. Nicolaenko, Global regularity of the $3 D$ Rotating Navier-Stokes Equations for resonant domains. Indiana Univ. Math. J., 48, (1999), 1133-1176.

2. J.-M. Bony, Calcul symbolique et propagation des singularités pour les équations aux dérivées partielles non linéaires. Ann. Sci. École Norm. Sup., 14, (1981), 209-246.

3. P. Constantin, The Littlewood-Paley Spectrum in Two-Dimensional Turbulence, Theo. Comput. Fluid Dynam., 9, (1997), 183-189.

4. P. E. Dimotakis, The mixing transition in turbulent flows, J. Fluid Mech., 409, (2000), 69-98.

5. U. Frisch, From global scaling, a la kolmogorov, to local multifractal scaling in fully developed turbulence, Proc. Roy. Soc. London A, 434, (1991), 89-99.

6. U. Frisch, Turbulence, Cambridge University Press, Cambridge, 1995.

7. S. Goto, A physical mechanism of the energy cascade in homogeneous isotropic turbulence, J. Fluid Mech., 605, (2008), 355-366.

8. S. Goto, Developed Turbulence: On the Energy Cascade, The Nihon Butsuri Gakkaishi (Butsuri), 73, (2018), 457-462.

9. S. Goto, Y. Saito, and G. Kawahara, Hierarchy of antiparallel vortex tubes in spatially periodic turbulence at high reynolds numbers, Phys. Rev. Fluids, 2, (2017), 064603.

10. S. Goto and J. C. Vassilicos, Local equilibrium hypothesis and taylor's dissipation law, Fluid Dyn. Res., 48, (2016), 021402.

11. T. Ishihara, T. Gotoh, and Y. Kaneda, Study of high-reynolds number isotropic turbulence by direct numerical simulation, Ann. Rev. Fluid Mech., 41, (2009), 165-180.

12. I.-J. Jeong and T. Yoneda, Vortex stretching and enhanced dissipation for the incompressible 3 D Navier-Stokes equations, Math. Annal., 380, (2021), 2041-2072.

13. R. R. Kerswell, Elliptical instability, Annu. Rev. Fluid Mech., 34, (2002), 83-113.

14. N. Kishimoto and T. Yoneda, Global solvability of the rotating Navier-Stokes equations with fractional Laplacian in a periodic domain, Math. Ann., 372, (2018), 743-779.

15. A. Kolmogorov, Local structure of turbulence in an incompressible fluid at very high reynolds number, Sov. Phys. Usp., 10, (1968), 734. 
16. R. McKeown, R. Ostilla-Mónico, A. Pumir, M. P. Brenner and S. M. Rubinstein, Turbulence generation through an iterative cascade of the elliptical instability, Sci. Adv., 6, (2020), eaaz2717.

17. Y. Motoori and S. Goto, Generation mechanism of a hierarchy of vortices in a turbulent boundary layer, J. Fluid Mech., 865, (2019), 1085-1109.

18. Y. Motoori and S. Goto, Hierarchy of coherent structures and real-space energy transfer in turbulent channel flow, J. Fluid Mech., 911, (2021), A27.

19. K. Ohkitani and S. Kida, Triad interactions in a forced turbulence, Phys. Fluids A, 4, (1992), 794-802.

20. S. A. Orszag and G. S. Patterson, Numerical simulation of three-dimensional homogeneous isotropic turbulence, Phys. Rev. Lett., 28, (1972), 76-79.

21. V. L. Rvačov and V. O. Rvačov, A certain finite function, Dopovidī Akad. Nauk Ukraïn. RSR Ser. A, 764, (1971), 705-707.

22. G. I. Taylor, Statistical theory of turbulence, Proc. Roy. Soc. A, 151, (1935), 421-444.

23. H. Tennekes and J. L. Lumley, A First Course in Turbulence, M.I.T. Press, Cambridge, Mass., 1972.

24. T. Tsuruhashi, S. Goto, S. Oka and T. Yoneda, Self-similar hierarchy of coherent tubular vortices in turbulence, to appear in Philosophical Trans. A.

25. J. C. Vassilicos, Dissipation in turbulent flows, Ann. Rev. Fluid Mech., 47, (2015), 95-114.

26. W. Volk, Evaluation of the solution of an integral-functional equation, J. Comput. Appl. Math., 174, (2005), 423-436.

27. M. Yamada and T. Yoneda, Resonant interaction of Rossby waves in two-dimensional flow on a beta plane, Physica D, 245, (2013), 1-7.

28. T. Yoneda, On the functional-differential equation of advanced type $f^{\prime}(x)=a f(2 x)$ with $f(0)=0$, J. Math. Anal. Appl., 317, (2006), 320-330.

29. T. Yoneda, On the functional-differential equation of advanced type $f^{\prime}(x)=a f(\lambda x), \lambda>1$, with $f(0)=0$, J. Math. Anal. Appl., 332, (2007), 487-496.

30. T. Yoneda, Long-time solvability of the Navier-Stokes equations in a rotating frame with spatially almost periodic large data, Arch. Ration. Mech. Anal., 200, (2011), 225-237.

Graduate School of Economics, Hitotsubashi University, 2-1 Naka, Kunitachi, Tokyo 186-8601, JAPAN

Email address: t.yoneda@r.hit-u.ac.jp

Graduate School of Engineering Science, Osaka University, 1-3 Machikaneyama, TOYONAKA, OSAKA 560-8531, JAPAN

Email address: s.goto.es@osaka-u.ac.jp

Graduate School of Mathematical Sciences, University of Tokyo, Komaba 3-8-1 MeGURO, TOKYO 153-8914, JAPAN

Email address: tomonori@ms.u-tokyo.ac.jp 